\title{
Antidepressant Prescribing Patterns in Korea: Results from the Clinical Research Center for Depression Study
}

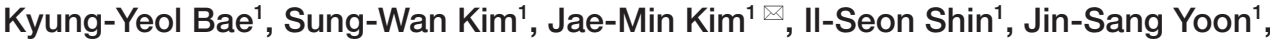 \\ Sung-Won Jung ${ }^{2}$, Min-Soo Lee ${ }^{3}$, Hyeon-Woo Yim ${ }^{4}$ and Tae-Youn Jun ${ }^{5}$ \\ 1'Department of Psychiatry, Chonnam National University Medical School, Gwangju, Korea \\ 2Department of Psychiatry, Keimyung University School of Medicine, Daegu, Korea \\ ${ }^{3}$ Department of Psychiatry, College of Medicine, Korea University, Seoul, Korea \\ ${ }^{4}$ Department of Preventive Medicine, The Catholic University of Korea College of Medicine, Seoul, Korea \\ ${ }^{5}$ Department of Psychiatry, The Catholic University of Korea College of Medicine, Seoul, Korea
}

Objective This study aimed to investigate antidepressant prescribing patterns, including initial choice, switching and combining, and concomitant use of non-antidepressant agents, for depressive disorders in naturalistic clinical care settings in Korea.

Methods Patients with depressive disorder were recruited from both outpatient and inpatient settings in 18 hospitals from all over Korea. Treatment was performed in naturalistic patterns based on each clinician's decision. Data were collected on the prescription of antidepressants and concomitant agents from baseline to 12-week follow-up.

Results Selective serotonin reuptake inhibitors (SSRIs) were the most commonly prescribed initial antidepressant (48.9\%), followed by newer dual-action antidepressants (45.8\%). When an SSRI was the initial antidepressant, $46.2 \%$ of patients whose medication was changed were moved to newer dual-action antidepressants, and $67.4 \%$ of combination cases were combined with newer dual-action ones. When a newer dual-action antidepressant was the initial antidepressant, $70.6 \%$ of patients whose medication was changed were moved to SSRIs, and other antidepressants including tricyclic antidepressants were most commonly added for combination treatment (50\% of combination cases). During the treatment period, $20.6 \%$ of antidepressants prescribed were augmented by non-antidepressant agents, and $75.1 \%$ were used concomitantly with anxiolytics or hypnotics. The most commonly used concomitant non-antidepressant agent was quetiapine.

Conclusion The selection of antidepressants and the concomitant use of non-antidepressant agents are becoming increasingly diversified, and the results of this study reflect changes in the prescribing pattern in actual Korean practices. Psychiatry Investig 2011;8:234-244

Key Words Antidepressive agents, Depression, Prescriptions, Korea, CRESCEND.

\section{INTRODUCTION}

Depressive disorders are highly prevalent throughout the world. ${ }^{1}$ Depressive disorders often impair social and occupational function and cause a considerable social burden. ${ }^{2-4}$ The optimal use of medications including antidepressants in the treatment of depressive disorders may reduce the social burden of depression as well as individual distress. For many years

Received: December 29, 2010 Revised: May 27, 2011

Accepted: May 29, 2011 Available online: August 26, 2011

$\triangle$ Correspondence: Jae-Min Kim, MD, PhD

Department of Psychiatry, Chonnam National University Medical School, 5 Hak-dong, Dong-gu, Gwangju 501-746, Korea

Tel: $+82-62-220-6143$, Fax: $+82-62-225-2351$

E-mail: jmkim@chonnam.ac.kr

(a) This is an Open Access article distributed under the terms of the Creative Commons Attribution Non-Commercial License (http://creativecommons.org/licenses/bync/3.0) which permits unrestricted non-commercial use, distribution, and reproduction in any medium, provided the original work is properly cited. after antidepressants were introduced for treatment for depression, tricyclic antidepressants (TCAs) were the first-line treatment of choice for depressive disorders. However, over the past decade, the first-line agent in the treatment of depression has shifted from TCAs to selective serotonin reuptake inhibitors (SSRIs). ${ }^{5,6}$ A number of previous reviews have found that TCAs and SSRIs have comparable efficacy but that SSRIs are generally better tolerated than TCAs. ${ }^{7,8}$ On the other hand, when newer antidepressants were recently introduced, they were expected to have superior efficacy compared with SSRIs because of their different mechanisms of action, including dual action or non-serotonergic action. A recent meta-analysis reported that serotonergic-noradrenergic antidepressants, so-called dual-action antidepressants, including venlafaxine and mirtazapine, seem to have a modest efficacy advantage over SSRIs. ${ }^{9}$ However, further systematic research is needed to 
confirm the superior efficacy of newer antidepressants in specific sub-populations of patients with depressive disorders or specific depressive symptoms. Despite the considerable development of antidepressants, recent studies suggested that many patients do not achieve a satisfactory outcome, and no single treatment strategy seems to be a panacea in the treatment of depressive disorders..$^{10}$ Obviously, there is a pressing need to develop more effective treatments for depression.

To enhance treatment success rates, the most appropriate antidepressants should be selected according to symptoms and patient characteristics. Many factors have been reported to influence the choice of antidepressant prescribed by clinicians, including the severity of depression, previous depressive episodes, the presence of comorbid conditions, and previous use of antidepressants. ${ }^{6,11-13}$ In cases of non-response or partial response, several treatment strategies are currently recommended as a next-step treatment, including switching to another antidepressant, combining two antidepressants, and using other agents concomitantly. ${ }^{12,14}$ Clinicians who treat patients with depression need to be kept abreast of the newer treatment strategies, and the need for a more understanding of these practices is clear.

Increasing numbers and types of antidepressant are now available in the Korean marketplace, so the armamentarium of antidepressant medications has expanded considerably. However, little is known about current prescribing practices in Korea in the treatment of depressive disorders. Most previous studies on antidepressant prescription practices in Korea have been in the form of survey research; although such studies might reflect preferred treatment strategies, they do not show real prescription patterns in actual clinical settings. To the best of our knowledge, there has been no nationwide prospective study on prescription patterns in the treatment of depressive disorders in actual practice settings in Korea.

The Clinical Research Center for Depression (CRESCEND) study is the first long-term prospective clinical study on depression in Korea, with a large nationwide sample population and government support. It is a naturalistic study in realworld practice settings investigating characteristics, courses of treatment, and outcomes in Korean patients with depressive disorders. Using the data from this study, the analyses presented here were carried out to investigate antidepressant prescribing patterns for the treatment of depressive disorders in real clinical settings over a 12 -week treatment period.

\section{METHODS}

\section{Study overview}

The design and procedure of the study have also been described elsewhere..$^{15}$ In total, 18 hospitals (16 university and 2 general hospitals) are collaborating in the ongoing CRESCEND study. The central coordinating center is located in the Psychiatric Department of the Catholic University Medical Center in Seoul and provides educational support and coordination for the enrollment and follow-up of study participants. The data-management center is in the Preventative Medicine Department of the Catholic University College of Medicine and provides support for obtaining data and quality control. Other regional centers located across South Korea play a role in recruitment and follow-up. With respect to the selection of regional centers, a nationwide distribution was sought, and at least two hospitals that were anticipated to be assessing and treating the largest numbers of people with depressive disorders (as opposed to hospitals primarily providing inpatient care for people with chronic psychotic disorders) were recruited from each province. Availability of personnel and study facilities were also taken into consideration. Enrolment took place in a naturalistic clinical environment and drew from both outpatient and inpatient settings, regardless of depression subtypes and physical comorbidity. Treatment interventions were also conducted in a naturalistic fashion with full autonomy for each clinician in determining the type, dose, and regimen of antidepressant and other medications. Assessments were scheduled at baseline, at 1, 2, 4, 8, 12, 24, and 52 weeks post-baseline, and annually thereafter. At each visit, a clinical review took place to decide treatment modalities. All other data on socio-demographic, clinical, and treatment-related characteristics were obtained by clinical research coordinators, who were trained and certified in clinical report form (CRF) implementation and data collection methods by the central coordinating center and supervised by the clinicians of the regional centers. Participants' data were recorded on a predetermined CRF at each visit, entered in the website homepage of the CRESCEND study (www.smileagain.or.kr) within two days, and monitored by personnel of the data-management center.

\section{Subjects}

All patients who visited the study hospitals seeking treatment for depression were candidate subjects for the study. Patients were assessed and diagnosed based on the Diagnostic and Statistical Manual of Mental Disorders, Fourth Edition $(\mathrm{DSM}-\mathrm{IV})^{16}$ criteria. Patients who were diagnosed with depressive disorders according to these diagnostic criteria were invited to participate in the study. All patients who agreed to take part in the study provided written informed consent. No advertising was used for the recruitment of subjects, and they received no economic compensation for study participation. The period for baseline recruitment was from January 2006 to August 2008. 
The CRESCEND study used broad inclusion criteria and minimal exclusion criteria to reflect real-world practices in Korea. Inclusion criteria were out- and inpatients aged over 7 years who were diagnosed with depressive disorders according to DSM-IV. Although there was no limitation to the baseline 17-item Hamilton Depression Rating Scale ${ }^{17}$ (HAMD) scores in the CRESCEND study, only patients with HAMD scores greater than 14 were included for the present analysis. Exclusion criteria were a history of comorbid DSM-IV diagnoses of psychotic disorders including schizophrenia, bipolar disorders, and dementia; any significant medical or neurological illnesses; and women who were currently pregnant or breastfeeding.

The study was approved by all relevant university and hospital institutional review boards. All participants reviewed the consent form, and written informed consent was obtained by research staff before participation in the study. For participants aged less than 16 years, written consent was obtained from a parent or legal guardian, and written assent was obtained from the participant. For those who were very old or physically ill, the nature and purpose of the study were explained, and written informed consent was obtained from the person or his/her caregiver, as appropriate.

\section{Socio-demographic and clinical characteristics}

Socio-demographic characteristics were evaluated by a clinical research coordinator using the structured CRF. Data were obtained on age, gender, duration of formal education, marital status, cohabiting status, religious observation, current occupation, and monthly income. For this analysis, the following categories were applied: marital status (currently married or not), cohabiting status (living alone or not), religion (religious observance or not), occupation (currently employed or not), and monthly income (above or below 2,000 USD).

As mentioned above, depressive disorder diagnoses were made by the treating clinicians based on DSM-IV criteria. In a sub-sample of patients who gave consent, the DSM-IV based Structured Clinical Interview ${ }^{18}$ (SCID) was administered for confirmation. Depressive disorders were divided into four groups: i) major depressive disorder without psychotic features, ii) major depressive disorder with psychotic features, iii) dysthymic disorder, and iv) depressive disorder not otherwise specified. The clinical research coordinators assessed all other clinical characteristics at baseline using the structured CRF: setting at enrollment (outpatient or inpatient), history of previous depressive episodes (recurrent or first episode), number of previous depressive episodes, age at onset and duration of illnesses, family history of depression, history of suicide attempt, and concurrent physical disorder. To determine suicide attempt history, participants were asked if suicide had ever been attempted, with supplementary questions about the method and severity of the attempt. Information on physical disorders was gathered using a questionnaire enquiring about 33 different disorders, which was recoded for this analysis as the presence or absence of any physical disorder.

Further assessment scales for evaluating symptoms and function were administered by the clinical research coordinators. The instruments administered were the HAMD, the Hamilton Anxiety Rating Scale ${ }^{19}$ (HAMA), the Clinical Global Impression Scale-severity scale ${ }^{20}$ (CGI-s), and the Social and Occupational Functioning Assessment Scale ${ }^{16}$ (SOFAS). All assessment scales have been formally translated and standardized in Korean. ${ }^{21-22}$

\section{Drug treatment}

Drug treatment was performed in naturalistic patterns based on each clinician's decision; that is, all clinicians who participated in this study themselves decided on the prescriptions of antidepressants and concomitant medications, considering each patient's condition. All types, regimens, and doses of antidepressants were allowed by the study. Clinicians decided on treatment modalities according to patients' states at each visit.

For the purpose of the analyses, antidepressants were classified as follows: SSRIs (citalopram/escitalopram, paroxetine, fluoxetine, sertraline); newer dual-action antidepressants (mirtazapine, venlafaxine, bupropion); other antidepressants including TCAs (milnacipran, imipramine, amitriptyline, nortriptyline, dothiepine, trazodone). Treatment regimens were classified into monotherapy, switching, and combination. Monotherapy was defined as using only one antidepressant during the treatment period. Switching was defined as switching from one to another antidepressant, and combination was defined as the use of two or more antidepressants for most of the treatment period.

Any concomitant medications used to enhance efficacy and relieve associated symptoms of depression or to control adverse effects were allowed, according to the clinicians' decision. These were classified into antipsychotics, mood stabilizers, and anxiolytics/hypnotics. Antipsychotics included risperidone, olanzapine, quetiapine, aripirazole, amisulpride, ziprasidone, perphenazine, haloperidol, and pimozide. Mood stabilizers were lithium and valproic acid, an anticonvulsant. Anxiolytics/hypnotics were lorazepam, alprazolam, diazepam, clonazepam, clorazepate, ethyl loflazepate, zolpidem, flunitrazepam, and bromazepam. Other concomitant agents such as buspirone were also prescribed.

\section{Statistical analyses}

The results described here are restricted to data obtained in 
the initial 12 weeks of treatment in order to focus on the acute treatment of depressive disorders. We present the results in number and percentage (\%) form for descriptive data using tables and figures.

We investigated the type of antidepressant(s) prescribed at the baseline visit and divided the treatment strategies used during the 12-week period of our investigation into monotherapy; switching, i.e., switching to another antidepressant; and combination, i.e., concomitant use of other agents besides antidepressants (augmentation) and concomitant use of antidepressants (combination). The use of anxiolytic/hypnotics during the study period was also investigated. In addition, information on the timing of switching, augmentation, and combination therapy was collected. These prescription patterns were compared again by the treatment setting, baseline HAMD and HAMA scores, and psychotic features using $\chi^{2}$ or
Fisher's exact tests.

\section{RESULTS}

\section{Patient sample and baseline characteristics}

A total of 1,183 patients were enrolled in the CRESCEND study, and data from 723 (61\%) patients were eligible for the present analysis; that is, these patients scored more than 14 on the HAMD and were taking antidepressants for up to 12 weeks, following the clinical decision of a clinician. Patients who had one or more visits after the baseline evaluation were included in the analysis. Patients disposition by hospitals and provinces was as follow: 181 at the Saint Mary's Hospital of the Catholic University, 243 at the Korea University Hospital, 28 at the Soonchunhyang University Hospital, 25 at the Hanyang University Hospital, and 2 at the Samsung Medical Cen-

Table 1. Demographic and clinical characteristics of the study population

Total sample $(\mathrm{N}=723)$

\begin{tabular}{|c|c|c|}
\hline \multicolumn{3}{|c|}{ Socio-demographic characteristics } \\
\hline \multicolumn{2}{|c|}{ Age, mean $(\mathrm{SD})$ years } & $48.4(16.0)$ \\
\hline \multicolumn{2}{|c|}{ Gender, N (\%) female } & $535(74.0)$ \\
\hline \multicolumn{2}{|c|}{ Education, mean $(\mathrm{SD})$ years } & $10.3(4.7)$ \\
\hline \multicolumn{2}{|c|}{ Marital status, $\mathrm{N}(\%)$ married } & $469(64.9)$ \\
\hline \multicolumn{2}{|c|}{ Living alone, $\mathrm{N}(\%)$} & $94(13.0)$ \\
\hline \multicolumn{2}{|c|}{ Religion, N (\%) have } & $424(58.6)$ \\
\hline \multicolumn{2}{|c|}{ Occupation, $\mathrm{N}(\%)$ no } & $466(64.5)$ \\
\hline \multicolumn{2}{|c|}{ Monthly income, $\mathrm{N}(\%)<2,000$ USD } & $356(49.2)$ \\
\hline \multicolumn{3}{|c|}{ Clinical characteristics } \\
\hline \multirow[t]{4}{*}{ Diagnosis } & Major depressive disorder without psychotic feature & $591(81.7)$ \\
\hline & Major depressive disorder with psychotic feature & $18(2.5)$ \\
\hline & Dysthymic disorder & $21(2.9)$ \\
\hline & Depressive disorder not otherwise specified & $93(12.9)$ \\
\hline \multicolumn{2}{|c|}{ Setting at enrollment, $\mathrm{N}(\%)$ outpatients } & $572(79.1)$ \\
\hline \multicolumn{2}{|c|}{ Recurrent depression, N (\%) } & $301(41.6)$ \\
\hline \multicolumn{2}{|c|}{ Number of depressive episodes, median (IQR) } & $1.0(1-2)$ \\
\hline \multicolumn{2}{|c|}{ Age at onset, mean (SD) years } & $44.7(17.2)$ \\
\hline \multicolumn{2}{|c|}{ Duration of illness, median (IQR) years } & $1.0(1-4)$ \\
\hline \multicolumn{2}{|c|}{ Family history of depression, N (\%) } & $99(13.7)$ \\
\hline \multicolumn{2}{|c|}{ History of suicide attempt, N (\%) } & $163(22.5)$ \\
\hline \multicolumn{2}{|c|}{ Physical disorders, $\mathrm{N}(\%)$ have } & $247(34.2)$ \\
\hline \multicolumn{3}{|c|}{ Assessment scales, mean (SD) scores } \\
\hline \multicolumn{2}{|c|}{ Hamilton Depression Rating Scale } & $21.6(4.9)$ \\
\hline \multicolumn{2}{|c|}{ Hamilton Anxiety Rating Scale } & $20.2(8.4)$ \\
\hline \multicolumn{2}{|c|}{ Clinical Global Impression-severity } & $4.8(1.0)$ \\
\hline \multicolumn{2}{|c|}{ Social and Occupational Functional Assessment Scale } & $57.1(11.0)$ \\
\hline
\end{tabular}

N: number, SD: standard deviation, USD: United States dollar, IQR: interquartile range 
ter located in Seoul; 39 at the Kangwon National University Hospital in Kangwon province; 89 at the Kyemyung University Hospital and 1 at the Kyungpook National University Hospital in Daegu; 1 at the Inje University Paik hospital and 1 at the Marynoll Hospital in Busan; 60 at the Chonnam National University Hospital and 12 at the Chosun University Hospital in Gwangju; and 41 at the Hwasun Chonnam National University Hospital in Jeollanam province.

Baseline socio-demographic and clinical characteristics of the patients are shown in Table 1. Most of the patients were female (74\%), were diagnosed as having major depressive disorder without psychotic features (82\%), and were enrolled from outpatient settings (79\%). Over half were believed to be first-episode cases. The patients included in our analysis $(n=723)$ did not differ from those excluded with respect to any sociodemographic or clinical characteristics (all p-values $>0.15$ ). However, the scores on all assessment scales suggested more severe symptoms or pathology in those included (all pvalues $<0.001$ ), reflecting our inclusion criterion of HAMD score $\geq 14$.

\section{Prescribing patterns}

\section{Choice of antidepressant at baseline}

Figure 1 shows the pattern of initial antidepressant choice. SSRIs were the most commonly prescribed initial antidepressant (48.9\% of all patients), followed by newer dual-action antidepressants ( $45.8 \%$ of all patients). The most commonly prescribed antidepressants among the SSRIs were escitalopram (22.4\%) and paroxetine (18.7\%), followed by fluoxetine $(4.1 \%)$ and sertraline (3.7\%). With regard to newer dual-action antidepressants, mirtazapine or venlafaxine is prescribed for

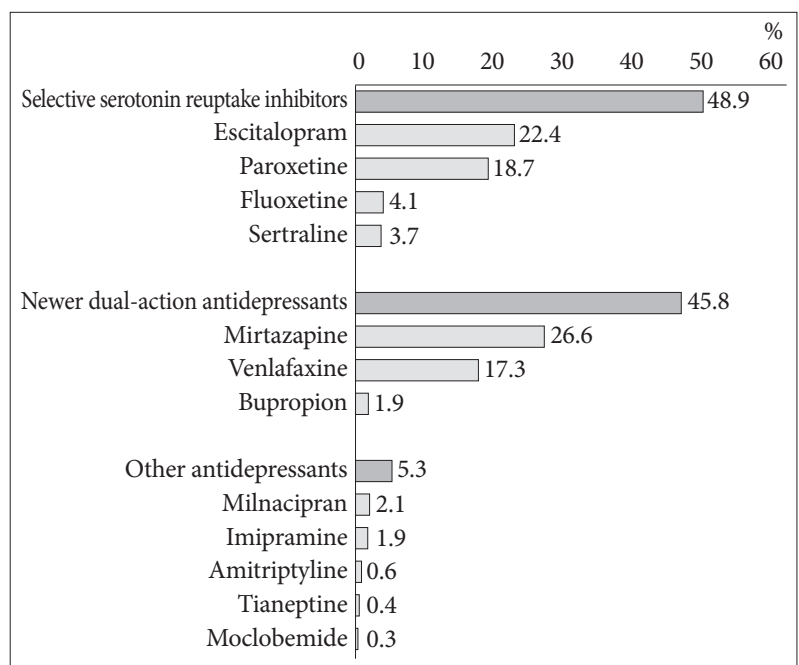

Figure 1. The prescribing pattern of antidepressants as first-line treatment.
$43.9 \%$ of all patients. Only $1.9 \%$ of patients were treated with bupropion. Other antidepressants including milnacipran, imipramine, amitriptyline, tianeptine, and moclobemide were initially prescribed for $5.3 \%$ of all patients.

With respect to prescribing patterns by the treatment setting, the initial antidepressants prescribed for inpatients were 48.3\% SSRIs, $45.7 \%$ newer dual-action antidepressants, and $6.0 \%$ other antidepressants; and for outpatients were $49.3 \%$ SSRIs, $45.8 \%$ newer dual-action antidepressants, $4.9 \%$ other antidepressants. There were no significant differences in the choice of initial antidepressant between the inpatients $(n=151)$ and outpatients $(n=572)(p=0.866)$. In addition, the HAMD and HAMA scores did not affect the choice of initial antidepressant [HAMD score $\geq 22$ ( $\mathrm{n}=324)$ vs. $<22(\mathrm{n}=399), \mathrm{p}=0.179$; and HAMA score $\geq 20(\mathrm{n}=339)$ vs. $<20(\mathrm{n}=384), \mathrm{p}=0.63$ ].

\section{Treatment pattern at the next step}

According to the data, regardless of the initial antidepressant prescribed, the largest proportion of patients received antidepressant monotherapy for most of the study period. Overall, $67.6 \%$ to $71.3 \%$ of patients were maintained on antidepressant monotherapy according to the initial antidepressants. Combination treatment with more than one antidepressant was used for $24.3 \%$ to $26.0 \%$ of patients. Switching from the initial antidepressant to another antidepressant occurred in $3.7 \%$ to $8.1 \%$ of patients.

\section{The pattern of antidepressant switching}

When an SSRI was initially prescribed, 38.5\% of patients were switched to other SSRIs, and $46.2 \%$ and $15.3 \%$ were switched to newer dual-action antidepressants and other antidepressants, respectively. When a newer dual-action or other antidepressant was the initial antidepressant, $70.6 \%$ of patients taking newer dual-action antidepressants and $66.7 \%$ of those taking other antidepressants, respectively, were switched to SSRIs; $23.5 \%$ of prescriptions for newer dual-action drugs were changed to another dual-action drug, and $33.3 \%$ of prescriptions for other medications were switched to a newer dual-action antidepressant. The proportion of patients switching from newer dual-action antidepressants to other antidepressants including TCAs was only 5.9\% (Figure 2). When the initial antidepressants were switched to non-SSRI agents, venlafaxine $(21.2 \%)$ was most commonly chosen among newer dual-action antidepressants, followed by mirtazapine (6.1\%), bupropion (6.1\%); switching to other antidepressants comprised only $9 \%$ of overall switching to non-SSRIs (milnacipran, 3\%; imipramine, 3\%; and amitriptyline, $3 \%$ ). Overall, the most commonly chosen switching strategy was switching to SSRIs (54.5\%), followed by switching to newer dual-action antidepressants (33.4\%) and to other antidepressants including 
TCAs (9\%). In terms of individual antidepressant, venlafaxine, a kind of serotonin/norepinephrine reuptake inhibitor (SNRI), was most commonly chosen (21.2\%), followed by SSRIs including escitalopram (18.2\%), paroxetine (15.2\%), and sertraline (15.2\%). There were no significant differences in the pattern of switching to individual antidepressants by the treatment setting (inpatient vs. outpatient, $\mathrm{p}=0.446$ ), the HAMD score (HAMD score $\geq 22$ vs. $<22, \mathrm{p}=0.137$ ) and HAMA score (HAMA score $\geq 20$ vs. $<20, \mathrm{p}=0.145$ ).

\section{The pattern of combination antidepressant treatment}

When SSRIs were initially prescribed, $67.4 \%$ of initial SSRIs were combined with newer dual-action antidepressants, and $22.5 \%$ and $10.1 \%$ were combined with other antidepressants including TCAs and other SSRIs, respectively. When newer dual-action antidepressants were the initial antidepressant,

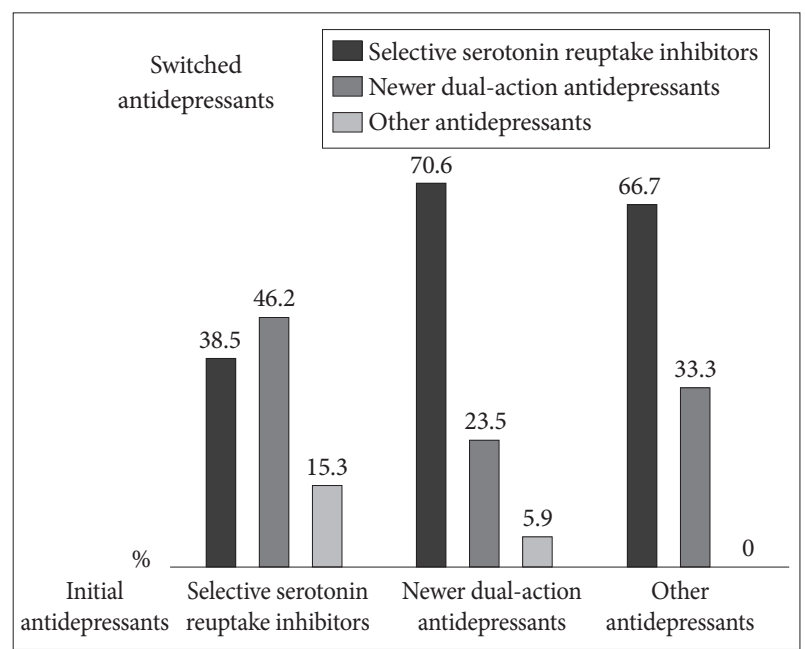

Figure 2. The switching pattern from the initial antidepressant to another one.

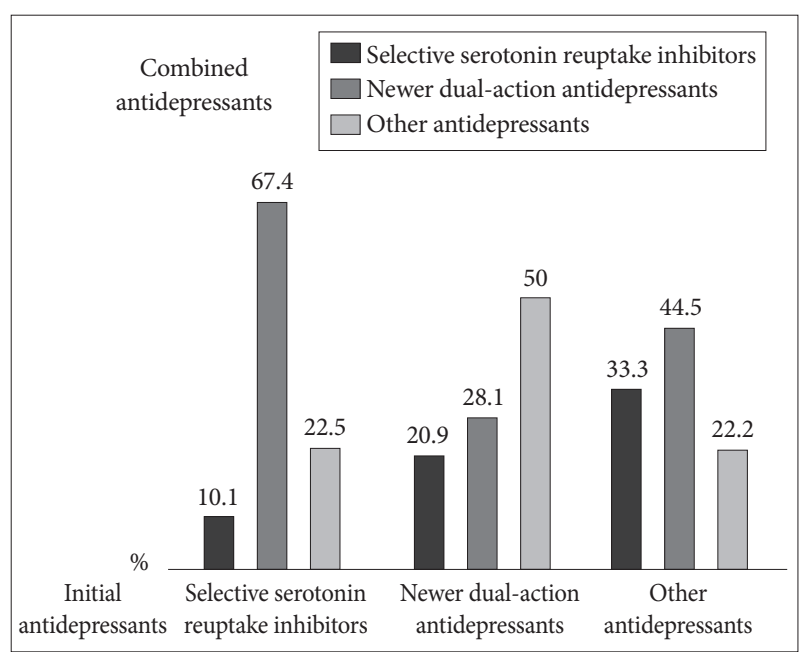

Figure 3. The pattern of combination antidepressant treatment according to the initial antidepressant. other antidepressants including TCAs were most commonly added for combination treatment (50\%), followed by other newer dual-action antidepressants (28.1\%) and SSRIs (20.9\%). When other antidepressants including TCAs were initially prescribed, newer dual-action antidepressants were most commonly added (44.5\%), followed by SSRIs (33.3\%) and other antidepressants including TCAs (22.2\%) (Figure 3). The most frequently used combination strategy was the newer dual-action antidepressants (47.8\%), followed by the other antidepressants including TCAs (35.9\%) regardless of the initial antidepressants. The antidepressant most frequently added to initiate combination therapy was amitriptyline (29.9\%), a TCA, followed by the newer dual-action antidepressants including mirtazapine (19.6\%), bupropion (17.9\%), and venlafaxine $(10.3 \%)$. There were no significant differences in the pattern of added antidepressants by the treatment setting (inpatient vs. outpatient, $\mathrm{p}=0.563$ ), the HAMD score (HAMD score $\geq 22$ vs. $<21, \mathrm{p}=0.379$ ) and HAMA score (HAMA score $\geq 20$ vs. $<20, \mathrm{p}=0.082$ ).

\section{The pattern of concomitant medication}

Of all prescriptions for antidepressants, $20.6 \%$ were augmented by other agents, and of these, $75.1 \%$ were used concomitantly with anxiolytics or hypnotics. The rates of concomitant medication according to the class of antidepressant are shown in Figure 4. The most frequently used concomitant medication was an antipsychotic agent (56.4\%), and quetiapine was the most frequently used of the available antipsychotic medications (29.5\%). Antipsychotic agents were given in 91 (12.9\%) depressive patients without psychotic features. Buspirone was frequently added to the antidepressant (28.2\%), and lithium (8.1\%) and anticonvulsants (7.4\%) were also frequent-

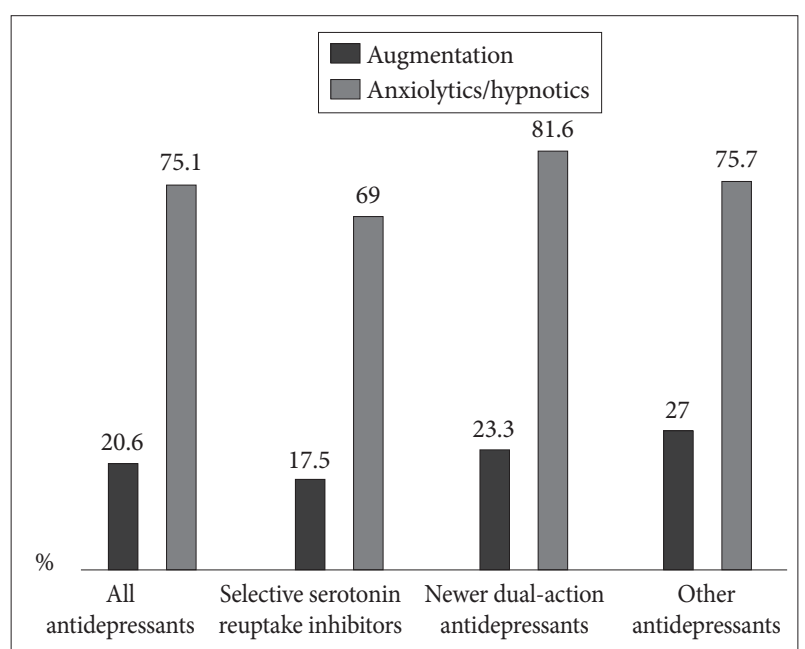

Figure 4. The concomitant use of non-antidepressant agents including anxiolytics/hypnotics according to the initial antidepressant. Augmentation agents include antipsychotics, lithium, and anticonvulsants. 
ly used as concomitant medications. In terms of the concomitant use of benzodiazepines, lorazepam (48.4\%) and alprazolam (36\%) were the benzodiazepines most frequently used concomitantly with the antidepressants. There were significant differences in the pattern of concomitant medication by the treatment setting. Lithium was more frequently prescribed in the inpatient setting (25.9\%) than the outpatient one (4.1\%) and buspirone was less frequently prescribed in the inpatient setting (7.4\%) than the outpatient one (32.8\%) (inpatient vs. outpatient, $\mathrm{p}<0.001)$. However, there were no significant differences in the pattern of concomitant medication by the HAMD score (HAMD score $\geq 22$ vs. $<21, \mathrm{p}=0.489$ ) and HAMA score (HAMA score $\geq 20$ vs. $<20, p=0.339$ ). The prescription rate of anxiolytics or hypnotics is higher in patients with HAMD score $\geq 22$ (80.9\%) compared to HAMD score $<21$ (70.4\%), $(\mathrm{p}=0.001)$.

\section{Timing of combination therapy, concomitant medication, and switching}

Most physicians (91\%) tended to use anxiolytics or hypnotics concomitantly from the beginning of antidepressant treatment. A considerable portion (56.4\%) of the concomitant use of other agents besides antidepressants started within 1 week of beginning treatment. However, $42.4 \%$ of antidepressant switching occurred after 2 weeks of treatment, and $36.4 \%$ and $21.2 \%$ of switching occurred after 3 and 4 weeks of treatment, respectively. With respect to initiation of combination treatment, $38 \%$ of such combinations were started during the first week of treatment, and $27.7 \%$ and $14.7 \%$ were started during weeks 2 and 3, respectively. Only 19.6\% of combination treatments were started during week 8 .

\section{DISCUSSION}

Data on the prescription of antidepressants in real-world psychiatric practice in Korea is limited. Most previous studies have used survey research or investigated the prescribing pattern in just one hospital. The CRESCEND study was planned to reflect the actual psychiatric clinical picture throughout Korea and its strength is that it is representative of Korea in terms of both sample size and the source of patients. To our knowledge, this study was the first nationwide prospective clinical study on Korean patients with depressive disorders. The results of this study show that many changes have occurred in Korean psychiatrists' prescribing of antidepressants.

A previous study in Korea, reported in 2003, found that SSRIs were preferred by many psychiatrists (70\%) as first-line agents for the treatment of major depressive disorder (MDD), and a much lower proportion of respondents $(8 \%)$ preferred newer dual-action antidepressants, including mirtazapine and venlafaxine. ${ }^{23}$ However, the results of the present study show that SSRIs were actually prescribed as first-line agents for only $48.9 \%$ of patients with MDD, and the rate of the prescription of newer dual-action antidepressants seems to have increased significantly, to $45.8 \%$. Mirtazapine was the most frequently prescribed antidepressant (26.6\%). Jung et al. ${ }^{23}$ suggested that newer dual-action antidepressants had not yet been proven safe and effective when they were introduced into Korea in the late 1990s, and therefore many psychiatrists preferred SSRIs, which had been demonstrated to be effective and safe agents. However, as Korean psychiatrists' cumulative experience in using newer dual-action antidepressants including mirtazapine has grown, prescription of these drugs has increased. In particular, mirtazapine is significantly less likely to cause the sexual dysfunction that SSRIs commonly cause and is also effective, without adjunctive benzodiazepine, against the decreased appetite, sleep problems, anxiety, and agitation frequently associated with MDD. ${ }^{24}$ In addition, a recent metaanalysis found that mirtazapine has a faster onset of action and higher remission rates than SSRIs in the first 6 weeks of treatment. These findings may explain the rapid increase in the use of mirtazapine as a first-line agent. ${ }^{25}$

The present study showed that the use of venlafaxine has also increased. A recent meta-analysis suggested that venlafaxine was associated with greater response (odds ratio 1.15) and remission (odds ratio 1.19) in the treatment of MDD, compared with SSRIs. ${ }^{26}$ The study showed that the odds ratio for response and remission was 1.35 in treatment-resistant depression, compared with SSRIs.

Although venlafaxine is known to be associated with sympathomimetic cardiovascular effects including hypertension and prolonged heart rate-corrected QT interval, ${ }^{27}$ the significant increase in the prescription of venlafaxine appears to reflect recent more aggressive trends in the prescription of antidepressants. Many studies and meta-analyses have suggested that dual-action antidepressants including venlafaxine and mirtazapine have superior efficacy over single-action antidepressants. ${ }^{28,29}$ Increased use of mirtazapine and venlafaxine in real practice seems to be related to the expectation of a better response.

With regard to switching, when the initial antidepressants were SSRIs, the most common switch was to newer dual-action antidepressants (46.2\%) followed by switching to other SSRIs (38.5\%). When the initial antidepressants were non-SSRIs, switching to SSRIs was most common (about 70\%). In terms of individual agents, switching to venlafaxine, a newer dual-action antidepressant, was most common (21.2\%), followed by SSRIs including escitalopram (18.2\%). The Korean Medication Algorithm for Major Depressive Disorder (KMAP- MD) suggests that switching from the initial antidepres- 
sant to venlafaxine and other SSRIs is reasonable in patients who failed to respond to the initial antidepressant. ${ }^{30}$ In particular, when the initial antidepressants are the newer dual-action types or TCAs, KMAP-MD recommends switching to SSRIs first, and this recommendation is concordant with the results of this study.

One notable finding of the present study concerns the timing of switching. Most current treatment guidelines for MDD recommend the continuous use of antidepressants for 4 to 8 weeks. ${ }^{11,1431}$ KMAP-MD also recommends that switching should be considered only after the continuous use of antidepressants for 3 to 5 weeks. The findings of this study showed that the actual mean switching time is $4.0 \pm 2.3$ weeks. However, a considerable proportion of switching ( $42.4 \%$ ) occurred within 2 weeks after initiation of treatment. This result is consistent with findings by Seo et al. ${ }^{32}$ who suggested that increased pressure for early discharge seems to be affecting treatment choices in inpatient settings. However, the findings of the present study show a similar trend in outpatient settings. A growing body of evidence suggests that response to antidepressant treatment in the first 2 weeks may be used as a predictor of subsequent outcomes. Therefore, it may be a useful strategy to switch antidepressants as early as possible instead of waiting for 4 to 8 weeks if patients do not respond quickly to treatment. ${ }^{33,34}$ A recent meta-analysis suggested that the early clinical response (i.e., 2 weeks) could be a predictor of the subsequent response and that there was little chance of a sustained response for patients who made no improvement within 2 weeks. ${ }^{35}$ In any case, the results of this study showed that switching time has been advanced in real practice, and systematic prospective studies in Korea are necessary to address this timing issue.

Among the patients in this study, 25\% received antidepressant combination treatment. The concomitant use of two antidepressants is controversial because of possible adverse effects and questionable clinical benefits. ${ }^{36}$ Current treatment guidelines for MDD usually recommend starting a single firstline agent and trying a series of other single agents if patients fail to respond to the first one. However, the findings of this study showed a gap between the guidelines and real practice. We observed that some psychiatrists started combination treatment from the early phase of treatment. In this study, 38\% of combination treatments started within 1 week of treatment commencing. The STAR*D (Sequenced Treatment Alternatives to Relieve Depression) study suggested that consecutive monotherapy cannot be the best treatment strategy for patients who seem not to respond to an initial monotherapy. ${ }^{37}$ Blier et al. ${ }^{38}$ reported that antidepressant combinations approximately doubled the remission rates achieved with single agent. In addition, NIMH (the National Institute of Mental
Health) recently funded a study, Combining Oral Medications to End Depression (COMED), to compare the potential benefits of antidepressant combination treatment at initiation of treatment. Evidence is accumulating that combination treatment from the very early phase of treatment may result in enhanced outcomes. Some researchers argue that this trend represents a major paradigm shift in the treatment of depression. ${ }^{39}$

In terms of the classes of antidepressants that may be combined, the present study found that when initial antidepressants were SSRIs, newer dual-action antidepressants were most frequently prescribed as concomitant antidepressants (67.4\%). A previous study to investigate the general practice of psychiatrists reported that SSRI plus mirtazapine was the most popular first-choice combination. ${ }^{40}$ That study suggested that psychiatrists chose this option because of the anxiolytic and sleep-inducing effects, dual-action mechanism, good interaction profile, rapid onset of action, and lack of typical SSRI side effects. The results of the present study also showed that, among newer dual-action antidepressants, mirtazapine was most commonly prescribed when a combination treatment was being considered. Another study on treatment given in a Korean university hospital reported that a combination treatment of an SSRI plus a TCA was most frequently chosen in 2001, but that in 2006, an SSRI plus mirtazapine was the most frequent combination, and a combination of mirtazapine and venlafaxine was often used in addition to the combination of an SSRI plus a TCA. ${ }^{32}$ However in the present study, bupropion was also frequently added to initial antidepressants (17.9\%). Bupropion is an antidepressant with properties of norepinephrine- and dopamine-reuptake inhibition instead of serotonergic action. The combination of bupropion and an SSRI or SNRI is widely used as an option that has a triple reuptake inhibitor-like action, and bupropion also seems to reverse certain adverse effects associated with serotonergic agents and to enhance the efficacy of initial antidepressants. ${ }^{41,42}$ On the other hand, in this study when the initial antidepressants were newer dual-action and other antidepressants including TCAs, other (50\%) and newer dual-action antidepressants (44.5\%), respectively, were frequently chosen as the concomitant antidepressant. That is, the combination of newer dual-action antidepressants plus other antidepressants including TCAs was commonly used.

Interestingly, however, amitriptyline was the most commonly used concomitant antidepressant (29.9\%). Jung et al. ${ }^{23}$ reported that $90 \%$ of psychiatrists who responded to a survey were prescribing amitriptyline in 2003. Amitriptyline has multiple pharmacological actions. In addition to noradrenergic and serotonergic actions, amitriptyline also has some affinity for muscarinic and histaminergic systems. ${ }^{43}$ Although some 
evidence indicates that amitriptyline has some adverse effects, it was most frequently prescribed as a first-line antidepressant in the past and was still the most commonly used concomitant antidepressant in this study. But the dose of amitriptyline was significantly lower (mean $12.3 \mathrm{mg} /$ day) in this study than that usually used for the treatment of depressive disorders. This result suggests that the amitriptyline was being used to control associated symptoms such as sleep disorder, pain, and agitation rather than to manage the depression itself. In general, the results of this study showed that combination strategies have diversified as new dual-action antidepressants have been introduced into the market.

Augmentation treatment with non-antidepressant agents can be used to accelerate treatment response. We found that about $20 \%$ of patients received augmentation treatment and the most commonly used augmenting agent was an antipsychotic. Atypical antipsychotic agents (52.3\%) were more frequently prescribed than were typical antipsychotics (4.1\%). Growing evidence suggests that other atypical antipsychotics are also associated with antidepressant effects through $5-\mathrm{HT}_{2}$ receptor antagonism and $5-\mathrm{HT}_{1 \mathrm{~A}}$ and dopamine receptor partial agonistic activity. ${ }^{44}$ Among the atypical antipsychotic agents in this study, quetiapine was the most commonly used (29.5\%). This result is concordant with the result of a previous study. Seo et al. ${ }^{32}$ suggested that an antidepressant effect of quetiapine started to receive attention because the effect of quetiapine on bipolar depression was demonstrated, and its use for this purpose was approved by the FDA (Food and Drug Administration). Quetiapine enhances central serotonergic transmission through $5-\mathrm{HT}_{2 \mathrm{~A}}$ receptor antagonism, and $5-\mathrm{HT}_{1 \mathrm{~A}}$ receptor modulation is thought to be important in quetiapine's antidepressant efficacy. ${ }^{45,46}$ Dopamine transmission is known to be enhanced in patients who are treated with quetiapine by antagonism of $5-\mathrm{HT}_{2 \mathrm{~A}}$ and partial agonistic activity at $5-\mathrm{HT}_{1 \mathrm{~A} .}{ }^{47,48} \mathrm{In}$ addition, previous studies have suggested that quetiapine may exert its antidepressant effect through effects on the glutamate receptor and norepinephrine transporter. ${ }^{45}$ It has also been reported that concomitant use of quetiapine in the treatment of depression improves sleep disturbance and causes earlier onset of antidepressant action, which may ultimately lead to the reduction of suicide risk. ${ }^{49}$ Other than quetiapine, aripiprazole has recently become the first atypical antipsychotic agent to be approved by the FDA for the adjunctive treatment of MDD. It is thought that atypical antipsychotics will play a greater role in the treatment of depression in the future.

This study showed that buspirone accounted for $28.2 \%$ of all concomitant use of agents in the "other antidepressant" category. Buspirone was first proposed for use in an augmentation strategy in the 1990s, but a controlled study found that the concomitant use of buspirone was no more effective than the addition of a placebo..$^{50}$ Buspirone augmentation looked promising in the STAR*D study, but the findings were unclear because the patient group who received buspirone augmentation had lower depressive symptoms at baseline. ${ }^{51}$ Further systematic study is needed to demonstrate the ultimate efficacy of buspirone augmentation.

In another paper from the same dataset of our study group, we investigated the relationship between a history of a suicide attempt and treatment characteristics. In brief, we did not find significant difference between patients with and without a history of a suicide attempt in the choice of antidepressant or the use of concomitant medications including antipsychotic agent. However, mood stabilizers were more frequently prescribed in patients with a history of a suicide attempt. ${ }^{52}$

In terms of anxiolytics and hypnotics including benzodiazepine, we found that about $75 \%$ of patients received benzodiazepine. A combination of antidepressant and benzodiazepine appears to be common practice throughout the world. However, the advantages of this combination treatment are unclear. Fava et al. ${ }^{53}$ recently reported that patients with MDD who were treated with fluoxetine plus the hypnotic eszopiclone showed more improvement on other depressive symptom scores and on sleep scores than did patients treated with fluoxetine alone. The study also found that patients treated with both an antidepressant and hypnotic agent showed significantly increased remission rates. The sleep-improving effects of hypnotics are suspected to enhance antidepressant efficacy. ${ }^{39}$ The Cochrane review also reported that combination treatment with both antidepressant and benzodiazepine is more likely to result in a positive response than is treatment with antidepressant alone. ${ }^{54}$ Therefore, the benefits of adding a benzodiazepine to antidepressant treatment should be balanced judiciously against possible risks, including dependence and accident proneness.

This study has some limitations. Firstly, this is an open-label naturalistic study for the treatment of depressive patients. The naturalistic design was both a strength and potential limitation. The broad inclusion and minimal exclusion criteria for recruitment, and the absence of limitations placed on treatment were designed to reflect real clinical situations as closely as possible and maximize generalizability to clinical practice. However, because the treatment modality was determined by the choice of the treating clinician rather than by any formal guideline, inter-clinician variability might affect observed outcomes. Secondly, most patients were recruited from the university hospitals (16 of 18 hospitals), which can affect the prescription patterns. Lastly, approximately three-fourths of patients were enrolled from outpatient settings. There are possibilities that patients who were enrolled were less severe and 
treatment setting affected the prescription patterns. However, we found that there were no significant differences in the prescription patterns according to the treatment setting (inpatient or outpatient).

Overall, the CRESCEND study, a nationwide naturalistic study, has several important implications for clinicians. Firstly, there is a gap between current treatment guidelines and realworld practice. The results of this study show the real trends of prescription practice in Korea, namely, that the choice of antidepressants and the concomitant use of other agents have become increasingly diversified as newer agents, such as dual-action antidepressants and atypical antipsychotics, have been introduced into Korea. The concomitant use of antidepressants and atypical antipsychotics including quetiapine has been increasing. In addition, the concomitant use of other agents seems to start from the early phase of treatment, and an early switching strategy is commonly used. Further systematic studies are required to develop new treatment guidelines for depression that are suited to Korean society today, that reflect changes in prescribing patterns in real practice, and that consider new research evidence in the treatment of depression. In addition, further studies to compare the results of this study with those of the National Health Insurance database will be useful to examine the actual Korean antidepressants prescription pattern.

\section{Acknowledgments}

This research was supported by a grant of the Korea Health 21 R\&D, Ministry of Health and Welfare, Republic of Korea (A050047, PI: TY Jun).

\section{REFERENCES}

1. Kessler RC, McGonagle KA, Zhao S, Nelson CB, Hughes M, Eshleman $\mathrm{S}$, et al. Lifetime and 12-month prevalence of DSM-III-R psychiatric disorders in the United States. Results from the National Comorbidity Survey. Arch Gen Psychiatry 1994;51:8-19.

2. Wells KB, Sherbourne CD. Functioning and utility for current health of patients with depression or chronic medical conditions in managed, primary care practices. Arch Gen Psychiatry 1999;56:897-904.

3. Hirschfeld RM, Montgomery SA, Keller MB, Kasper S, Schatzberg AF, Möller HJ, et al. Social functioning in depression: a review. J Clin Psychiatry 2000;61:268-275.

4. Kim JM, Stewart R, Kim SW, Yang SJ, Shin IS, Kim YH, et al. Interactions between life stressors and susceptibility genes (5-HTTLPR and BDNF) on depression in Korean elders. Biol Psychiatry 2007;62:423428 .

5. Lawrenson RA, Tyrer F, Newson RB, Farmer RD. The treatment of depression in UK general practice: selective serotonin reuptake inhibitors and tricyclic antidepressants compared. J Affect Disord 2000;59:149157.

6. Anderson IM, Ferrier IN, Baldwin RC, Cowen PJ, Howard L, Lewis G, et al. Evidence-based guidelines for treating depressive disorders with antidepressants: a revision of the 2000 British Association for Psychopharmacology guidelines. J Psychopharmacol 2008;22:343-396.

7. Anderson IM. Selective serotonin reuptake inhibitors versus tricyclic antidepressants: a meta-analysis of efficacy and tolerability. J Affect Disord 2000;58:19-36.
8. MacGillivray S, Arroll B, Hatcher S, Ogston S, Reid I, Sullivan F, et al. Efficacy and tolerability of selective serotonin reuptake inhibitors compared with tricyclic antidepressants in depression treated in primary care: systematic review and mbeta-analysis. BMJ 2003;326:1014.

9. Papakostas GI, Thase ME, Fava M, Nelson JC, Shelton RC. Are antidepressant drugs that combine serotonergic and noradrenergic mechanisms of action more effective than the selective serotonin reuptake inhibitors in treating major depressive disorder? A meta-analysis of studies of newer agents. Biol Psychiatry 2007;62:1217-1227.

10. Wisniewski SR, Fava M, Trivedi MH, Thase ME, Warden D, Niederehe $\mathrm{G}$, et al. Acceptability of second-step treatments to depressed out patients: a STAR*D report. Am J Psychiatry 2007;164:753-760.

11. American Psychiatric Association. Practice guideline for the treatment of patients with major depressive disorder (revision). Am J Psychiatry 2000;157:1-45.

12. Bauer M, Bschor T, Pfennig A, Whybrow PC, Angst J, Versiani M, et al. World Federation of Societies of Biological Psychiatry (WFSBP) Guidelines for Biological Treatment of Unipolar Depressive Disorders in Primary Care. World J Biol Psychiatry 2007;8:67-104.

13. Bauer M, Monz BU, Montejo AL, Quail D, Dantchev N, Demyttenaere $\mathrm{K}$, et al. Prescribing patterns of antidepressants in Europe: results from the Factors Influencing Depression Endpoints Research (FINDER) study. Eur Psychiatry 2008;23:66-73.

14. Bauer M, Whybrow PC, Angst J, Versiani M, Moller HJ; World Federation of Societies Biological Psychiatry Task Force on Treatment Guidelines for Unipolar Depressive Disorders. World Federation of Societies of Biological Psychiatry (WFSBP) Guidelines for Biological Treatment of Unipolar Depressive Disorders, Part 1: Acute and continuation treatment of major depressive disorder. World J Biol Psychiatry 2002;3:5-43.

15. Kim JM, Kim SW, Stewart R, Kim SY, Yoon JS, Jung SW, et al. Predictors of 12-week remission in a nationwide cohort of people with depressive disorders: the CRESCEND study. Hum Psychopharmacol 2011;26:41-50.

16. American Psychiatric Association. Diagnostic and Statistical Manual of Mental Disorders, Fourth edition. Washington DC: American Psychiatric Association; 1994.

17. Hamilton MA. A rating scale for depression. J Neurol Neurosurg Psychiatry 1960;23:56-62.

18. First MB, Gibbon M, Spitzer RL, Williams JBW. User's Guide for the Structured Clinical Interview for DSM-IV Axis I Disorders Research Version (SCID-I). New York, NY: New York State Psychiatric Institute, Biometrics Research; 1996.

19. Hamilton M. The assessment of anxiety states by rating. Br J Med Psychol 1959;32:50-55.

20. Guy W. ECDEU Assessment Manual for Psychopharmacology, Revised. Rockville, MD: National Institute of Mental Health; 1976.

21. Lee JY, Cho MJ, Kwon JS. Global assessment of functioning scale and social and occupational functioning scale. Korean J Psychopharmacol 2006;17:122-127.

22. Yi JS, Bae SO, Ahn YM, Park DB, Noh KS, Shin HK, et al. Validity and reliability of the Korean version of the Hamilton Depression Rating Scale (K-HDRS). J Korean Neuropsychiatr Assoc 2005;44:456-465.

23. Jung HY, Lee SI, Kim JK, Park JH. Treatment strategy for antidepressant-resistant depression in Korea: a survey of clinicians. Korean J Psychopharmacol 2003;14:40-47.

24. Watanabe N, Omori IM, Nakagawa A, Cipriani A, Barbui C, McGuire $\mathrm{H}$, et al. Safety reporting and adverse-event profile of mirtazapine described in randomized controlled trials in comparison with other classes of antidepressants in the acute-phase treatment of adults with depression: systematic review and meta-analysis. CNS Drugs 2010;24:3553.

25. Thase ME, Nierenberg AA, Vrijland P, van Oers HJ, Schutte AJ, Simmons JH. Remission with mirtazapine and selective serotonin reuptake inhibitors: a meta-analysis of individual patient data from 15 controlled trials of acute-phase treatment of major depression. Int Clin Psycho- 
pharmacol 2010;25:189-198.

26. Bauer M, Tharmanathan P, Volz HP, Moeller HJ, Freemantle N. The effect of venlafaxine compared with other antidepressants and placebo in the treatment of major depression: a meta-analysis. Eur Arch Psychiatry Clin Neurosci 2009;259:172-185.

27. Howell C, Wilson AD, Waring WS. Cardiovascular toxicity due to venlafaxine poisoning in adults: a review of 235 consecutive cases. Br J Clin Pharmacol 2007;64:192-197.

28. Stahl SM, Entsuah R, Rudolph RL. Comparative efficacy between venlafaxine and SSRIs: a pooled analysis of patients with depression. Biol Psychiatry 2002;52:1166-1174.

29. Gutierrez MA, Stimmel GL, Aiso JY. Venlafaxine: a 2003 update. Clin Ther 2003;25:2138-2154.

30. Seo JS, Min KJ, Kim W, Seok JH, Bahk WM, Song HC, et al. Korean medication algorithm for depressive disorder 2006 (I). J Korean Neuropsychiatr Assoc 2007;46:453-460.

31. National Institute for Clinical Excellence. Depression: Management of Depression in Primary and Secondary Care. Clinical Guideline 23. London: National Institute for Clinical Excellence; 2004.

32. Seo HJ, Jung YE, Lee J, Chae JH, Jun TY, Bahk WM. Changes of medication usage in inpatients with major depressive disorder: one university hospital between year 2001 and 2006. Korean J Psychopharmacol 2007;18:399-407.

33. Nakajima S, Suzuki T, Watanabe K, Kashima H, Uchida H. Accelerating response to antidepressant treatment in depression: a review and clinical suggestions. Prog Neuropsychopharmacol Biol Psychiatry 2010;34: 259-264.

34. Katz MM, Tekell JL, Bowden CL, Brannan S, Houston JP, Berman N, et al. Onset and early behavioral effects of pharmacologically different antidepressants and placebo in depression. Neuropsychopharmacology 2004;29:566-579.

35. Szegedi A, Jansen WT, vanWilligenburg AP, van der Meulen E, Stassen $\mathrm{HH}$, Thase ME. Early improvement in the first 2 weeks as a predictor of treatment outcome in patients with major depressive disorder: a metaanalysis including 6562 patients. J Clin Psychiatry 2009;70:344-353.

36. Horgan D, Dodd S, Berk M. A survey of combination antidepressant use in Australia. Australas Psychiatry 2007;15:26-29.

37. Rush AJ. Limitations in efficacy of antidepressant monotherapy. J Clin Psychiatry 2007;68 (suppl 10):8-10.

38. Blier P, Ward HE, Tremblay P, Laberge L, Hébert C, Bergeron R. Combination of antidepressant medications from treatment initiation for major depressive disorder: a double-blind randomized study. Am J Psychiatry 2010;167:281-288.

39. Stahl SM. Combining antidepressant therapies from the initiation of treatment: a paradigm shift for major depression. J Clin Psychiatry 2009; 70:1493-1494.

40. de la Gándara J, Agüera L, Rojo JE, Ros S, de Pedro JM. Use of antidepressant combinations: which, when and why? Results of a Spanish survey. Acta Psychiatr Scand Suppl 2005;428:32-36.
41. Zisook S, Rush AJ, Haight BR, Clines DC, Rockett CB. Use of bupropion in combination with serotonin reuptake inhibitors. Biol Psychiatry 2006;59:203-210.

42. Bodkin JA, Lasser RA, Wines JD Jr, Gardner DM, Baldessarini RJ. Combining serotonin reuptake inhibitors and bupropion in partial responders to antidepressant monotherapy. J Clin Psychiatry 1997;58: 137-145.

43. Garattini S, Samanin R. Biochemical hypotheses on antidepressant drugs: a guide for clinicians or a toy for pharmacologists? Psychol Med 1988; 18:287-304.

44. DeBattista C, Hawkins J. Utility of atypical antipsychotics in the treatment of resistant unipolar depression. CNS Drugs 2009;23:369-377.

45. Pae CU, Sohi MS, Seo HJ, Serretti A, Patkar AA, Steffens DC, et al. Quetiapine XR: current status for the treatment of major depressive disorder. Prog Neuropsychopharmacol Biol Psychiatry 2010;34:11651173.

46. Figueroa C, Brecher M, Hamer-Maansson JE, Winter H. Pharmacokinetic profiles of extended release quetiapine fumarate compared with quetiapine immediate release. Prog Neuropsychopharmacol Biol Psychiatry 2009;33:199-204.

47. Yatham LN, Goldstein JM, Vieta E, Bowden CL, Grunze H, Post RM, et al. Atypical antipsychotics in bipolar depression: potential mechanisms of action. J Clin Psychiatry 2005;66(Suppl 5):40-48.

48. Ichikawa J, Ishii H, Bonaccorso S, Fowler WL, O'Laughlin IA, Meltzer HY. 5-HT(2A) and $\mathrm{D}(2)$ receptor blockade increases cortical DA release via $5-\mathrm{HT}(1 \mathrm{~A})$ receptor activation: a possible mechanism of atypical antipsychotic-induced cortical dopamine release. J Neurochem 2001;76: 1521-1531.

49. Rihmer Z. Antidepressive efficacy of quetiapine XR in unipolar major depression--the role of early onset of action and sleep-improving effect in decreasing suicide risk. Neuropsychopharmacol Hung 2009;11:211215.

50. Landen M, Björling G, Agren H, Fahlen T. A randomized, double-blind, placebo-controlled trial of buspirone in combination with an SSRI in patients with treatment-refractory depression. J Clin Psychiatry 1998; 59:664-668.

51. Carvalho AF, Machado JR, Cavalcante JL. Augmentation strategies for treatment-resistant depression. Curr Opin Psychiatry 2009;22:7-12.

52. Kim SW, Stewart R, Kim JM, Shin IS, Yoon JS, Jung SW, et al. Relationship between a history of a suicide attempt and treatment outcomes in patients with depression. J Clin Psychopharmacol 2011;31:449-456.

53. Fava M, McCall WV, Krystal A, Wessel T, Rubens R, Caron J, et al. Eszopiclone co-administered with fluoxetine in patients with insomnia coexisting with major depressive disorder. Biol Psychiatry 2006;59: 1052-1060.

54. Furukawa TA, Streiner DL, Young LT. Antidepressant and benzodiazepine for major depression. Cochrane Database Syst Rev 2002;1: CD001026. 